\title{
Growth hormone and prolactin stimulation by Madopar in Parkinson's disease
}

\author{
A MARTINEZ-CAMPOS,* P GIOVANNINI, E PARATI, A NOVELLI, \\ T CARACENI, EE MÚLLER
}

From the Department of Pharmacology, University of Milan and Istituto Neurologico C Besta, Milan, Italy

SUMMARY Madopar, a combination of levodopa with benserazide, induced an inconsistent rise in plasma growth hormone in unmedicated patients with Parkinson's disease and in controls, and a greater growth hormone rise in Parkinsonian subjects on chronic Madopar therapy. In subjects on chronic therapy with levodopa and carbidopa (Sinemet), the growth hormone releasing effect of Madopar was blunted. Madopar increased plasma prolactin (PRL) in controls, unmedicated patients and patients on Madopar therapy while in patients on Sinemet therapy the PRL-releasing effect of Madopar was strikingly reduced. Since these data were interpreted as due to a defective dopamine tone in the hypothalamus of Parkinsonian subjects on Madopar but not Sinemet therapy, a direct dopamine receptor agonist, lisuride was administered. Lisuride, however, elicited a blunted growth hormone response both in patients on Madopar and Sinemet therapy, without revealing a state of supersensitivity of dopamine receptors for growth hormone control in Parkinsonian subjects on Madopar therapy. No difference was present in the PRL-lowering effect of lisuride in the different experimental groups. These findings suggest that: (1) hypothalamic dopamine function is impaired in Parkinsonian subjects on Madopar therapy, preserved in unmedicated patients and enhanced in patients on Sinemet therapy; (2) the endocrine effects observed in Parkinsonian subjects on chronic Madopar therapy may be due to some penetration of benserazide across the blood brain barrier in the region of the hypothalamus; (3) since Madopar and Sinemet are in essence equally effective antiparkinsonian remedies, penetration of benserazide does not occur across the blood brain barrier surrounding the nigrostriatal system.

Inhibitors of the L-aromatic amino acid decarboxylase (L-AAAD), the enzyme that converts the aromatic amino acids into the corresponding amine neurotransmitters decrease the concentrations of monoamine in various tissues while increasing the concentration of administered aromatic amino acids in blood. ${ }^{2}$ Among L-AAAD inhibitors, both carbidopa (MK-846, Merck Sharp \& Dohme) and benserazide (Ro 4-4602, Hoffmann La Roche) are widely used compounds. Reportedly, they do not cross in effective amounts the blood brain barrier

*Conacyt post-doctoral fellow, on leave from the Hospital Universitario "Dr José E Gonzales", Monterrey, NL Mexico. Present address: Unidad de Medicina Bio Psico Social, Hospital General de Mexico, SSA, Dr Balmis 148, Mexico 7, DF.

Address for reprint requests: Dr EE Müller, Department of Pharmacology, University of Milan, Via Vanvitelli, 32-20129 Milan, Italy.

Accepted 1 July 1981
(BBB), when given in a limited dose range; hence they are considered mainly peripheral inhibitors of L-AAAD, with the ability to prevent in vivo the conversion of aromatic amino acids in peripheral tissues but not in the brain. ${ }^{2}$ As a result, higher amounts of the unmetabolised amino acid are shunted to the central nervous system (CNS) where it can be converted to dopamine (DA). These premises formed the rationale for the use of inhibitors of L-AAAD that is carbidopa and benserazide, in conjunction with levodopa in the treatment of Parkinson's disease. Treatment of Parkinsonian subjects has progressed considerably with the combined use of levodopa and L-AAAD inhibitors, which have led to a marked reduction in levodopa doses whilst maintaining cerebral levels of DA unchanged. ${ }^{3}$

Dopamine present in the tuberoinfundibular (TI) region of the hypothalamus is a neurotransmitter 1116 
which plays a key role in neuroendocrine regulation; it can be released directly into the hypophyseal portal circulation to inhibit prolactin (PRL) secretion from pituitary lactotrophs or may act directly on CNSDA synapses to stimulate growth hormone $(\mathrm{GH})$ secretion. As a corollary, levodopa and other DAmimetic drugs are potent PRL-lowering and GHstimulant drugs in man (see ref 4 , for review).

Inhibitors of L-AAAD administered with levodopa, by shunting higher amounts of the amino acid into the CNS would be expected to exacerbate DAmediated neuroendocrine effects. In fact, in the human the addition of carbidopa with an oral dose of levodopa resulted in a fivefold augmentation of plasma levodopa and a twofold increase in GH levels. ${ }^{5}$ The GH stimulation induced by levodopacarbidopa (Sinemet) is so consistent that this combination is used as a screening test for $\mathrm{GH}$ deficiency in man. ${ }^{6}$

We report here a study conducted in Parkinsonian subjects, in which we investigated the ability of levodopa associated to the other L-AAD inhibitor benserazide (Madopar) to affect GH and PRL secretion. Previous studies had shown that some differences exist in the neuroendocrine effects of carbidopa and benserazide; while carbidopa only slightly affect baseline PRL levels, ${ }^{7} 8$ administration of a single dose of benserazide is followed by striking rises in plasma PRL. ${ }^{8}$ In addition, since the study with Madopar had provided results we thought of interest, we decided to investigate in some Parkinsonian patients the neuroendocrine effects of a direct DA receptor agonist, lisuride. ${ }^{10}$

\section{Materials and methods}

Fifty-six subjects, 39 patients and 17 controls were investigated with their informed consent. Patients comprised 31 women and 8 men, aged 37-69 years (mean 57.4) with idiopathic Parkinson's disease of 1-11 years duration (mean 6.2). Control subjects comprised 13 women and 4 men aged 35-71 years (mean 47.0). They were in-patients who volunteered for the study, recovered from minor disease, with no family history of diabetes and free of endocrine and metabolic disorders.

\section{Madopar studies}

Unmedicated patients Five women and 1 man aged 37-62 years (mean 47.2) with idiopathic Parkinsonism of 2-7 years duration (mean $3 \cdot 7$ years).

Patients on chronic Madopar therapy Six women aged 42-74 years (mean 54.2) with idiopathic Parkinsonism of 2-10 years duration (mean 5.0), who were taking one capsule of Madopar (125 mg) two to six times daily for periods ranging from 6 months to 6 years.

Patients on chronic Sinemet therapy Five women and one man aged 61-76 years (mean 68.0) with idiopathic
Parkinsonism of 9-11 years duration (mean 8.6), who were taking one capsule of Sinemet (levodopa $250 \mathrm{mg}$, carbidopa (L- $\alpha$-methyldopa hydrazine, $25 \mathrm{mg}$ ) one to three times daily for periods ranging from 1 to 6 years.

Patients withdrawn from therapy Five women and three men, aged 62-70 years (mean 66.5) with idiopathic Parkinsonism of 3-6 years duration (mean 4-1) had withdrawn Madopar therapy (see above) 4 days before; three men and one women, aged 45-68 years (mean 59.3) with idiopathic Parkinsonism of 4-6 years duration (mean 5.0) had withdrawn Sinemet therapy 4 days before. Eight women and two men, aged $42-71$ years (mean $48 \cdot 2$ ) served as controls in these studies.

\section{Lisuride studies}

Unmedicated patients Three women and one man aged 29-96 years (mean 47.6) with idiopathic Parkinsonism of 1-8 years duration (mean 3.6). One female patient of this group had been previously evaluated in the Madopar studies.

Patients on chronic Madopar therapy Six women aged 42-54 years (mean 50.0) with idiopathic Parkinsonism of 4-10 years duration (mean 6.4) who were taking one capsule of Madopar 3-6 times daily for periods ranging from 2 to 5 years. Two of these patients had been previously evaluated in the Madopar studies.

Patients on chronic Sinemet therapy Three women and two men, aged 52-76 years (mean 60.0) with idiopathic Parkinsonism of 5-11 years duration (mean 7.2), who were taking one capsule of Sinemet two to four times daily for periods ranging from 1 to 5 years. Three of these patients had been previously evaluated in the Madopar studies.

Patients withdrawn from Sinemet therapy These subjects comprised those of the preceding experimental group, and, in addition, one woman, aged 66 years, with Parkinsonism for 2 years, who had never been investigated. All subjects had withdrawn Sinemet therapy 4 days previously.

Five women and 2 men, aged 36-56 years (mean 46.5) served as controls in these studies.

\section{Clinical procedure}

All experiments were performed in the morning after an overnight fast and about 12 hours after the last drug administration. Informed consent was obtained from each patient. All subjects were supine, comfortable and free of stress during the test. Serial blood specimens were collected through an indwelling polyethylene catheter placed in the antecubital vein and kept open by a slow infusion of saline starting at 0800-0830 hours. After a 60 minute interval from placement of the venous catheter, one capsule of Madopar (200 mg levodopa) and $50 \mathrm{mg}$ $\mathrm{N}^{\prime}$ ((DL-seryl) $\mathrm{N}^{\prime \prime \prime}(2,3,4$-trihydroxybenzyl) hydrazine) or one tablet of lisuride $(0.2 \mathrm{mg}$, Lysenil, Schering) was administered and samples were obtained at $-30,0$ and $30,60,90,120,150$ and 180 minutes following drug administration. No side effects such as nausea, vomiting, 
hypotension etc, were observed in Parkinsonian subjects either during chronic treatment or when acutely challenged with Madopar. The same was true for controls receiving acute Madopar administration. In this respect, also administration of lisuride was ineffective in both patients and controls.

\section{Hormone assays}

In all experiments plasma was obtained by centrifugation and GH and PRL were assayed by double antibodyradioimmunoassay. The GH and PRL standards were NIH-GH-HS 20196 and NIH-PRL-VLS \#3, respectively; and the sensitivity of the assay was 0.1 and $0.3 \mathrm{ng} / \mathrm{ml}$, respectively. Doses up to $100 \mathrm{ng} / \mathrm{ml}$ of $\mathrm{GH}$ and PRL did not cross-react in the reciprocal assay. To avoid possible interassay variations, all samples of each experiment were assayed in a single radioimmunoassay. Data were expressed as absolute values $(\mathrm{ng} / \mathrm{ml} \pm \mathrm{SEM})$ and significance of difference was calculated by Dunnett's $t$ test.

\section{Results}

\section{MADOPAR STUDIES}

\section{Growth hormone}

Basal GH levels in unmedicated Parkinsonian subjects were not different from those present in controls $(0.5 \pm 0.1$ vs $0.6 \pm 0.1 \mathrm{ng} / \mathrm{ml})$; chronic treatment with Madopar did not affect basal GH levels $(0.7 \pm 0.1 \mathrm{ng} / \mathrm{ml}$ in patients on Madopar vs $0.6 \pm 0.1 \mathrm{ng} / \mathrm{ml}$ and $0.4 \pm 0.1 \mathrm{ng} / \mathrm{ml}$ in controls and

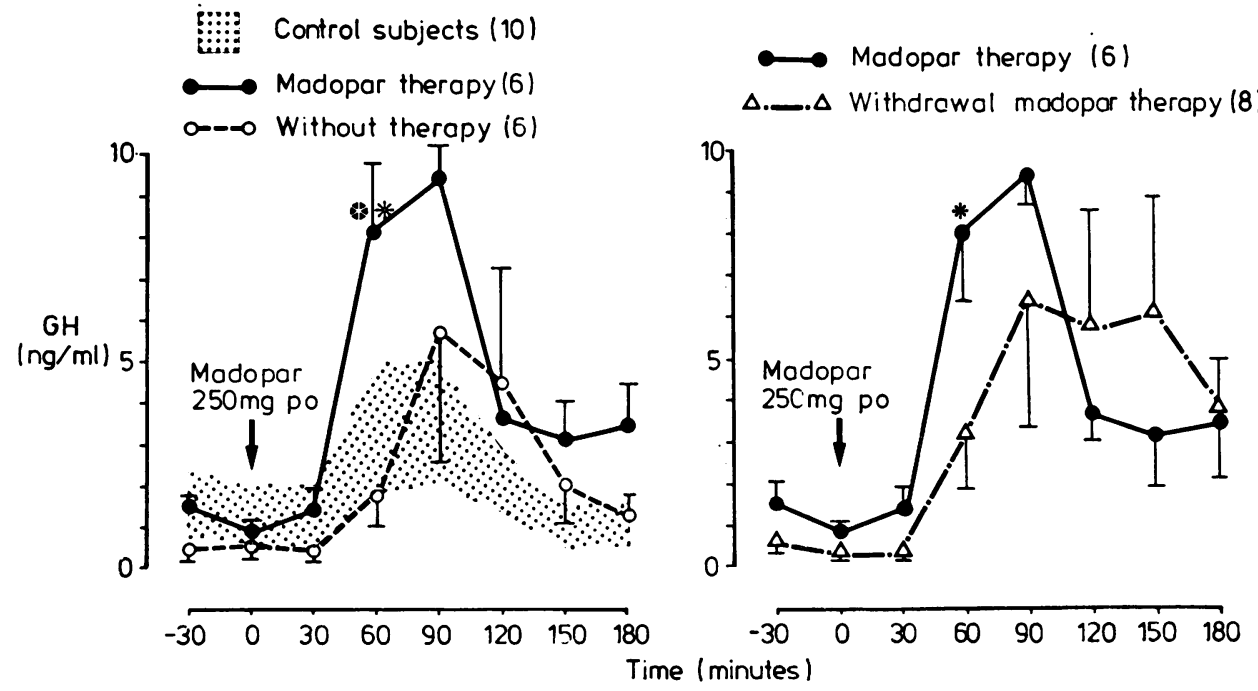

Fig 1 Left panel. Effect of Madopar on GH levels in control subjects, unmedicated Parkinsonian subjects and patients on Madopar therapy. Data are expressed as absolute values and SEM. Shaded area outlines the range of $\mathrm{GH}$ values after Madopar in control subjects. Number of subjects for each group are shown in parentheses. * = difference statistically significant vs controls: $*=$ difference statistically significance vs unmedicated PD subjects. Right panel. Effect of Madopar on plasma GH levels in patients on or withdrawn from Madopar therapy. *= difference statistically significant vs patients withdrawn from Madopar therapy. See text for further details. The same description applies to figs $2-4$.

in patients withdrawn from Madopar, respectively). Figure 1 (left panel) shows that acute administration of Madopar induced an inconsistent rise in plasma $\mathrm{GH}$ levels in controls (peak levels $3.4 \pm 1 \cdot 1$ and $3.5 \pm 1.6 \mathrm{ng} / \mathrm{ml}$ at 60 and 90 minutes, respectively). The GH-releasing effect of Madopar was instead clear-cut in Parkinsonian subjects on Madopar therapy; plasma $\mathrm{GH}$ peaked at 90 minutes $(9 \cdot 4 \pm 0.7$ $\mathrm{ng} / \mathrm{ml}$ ) and $\mathrm{GH}$ values different from control values were present at 60 minutes $(p<0.05)$. Unlike patients on Madopar therapy, the effect of Madopar on GH release in previously unmedicated subjects was not different from that present in controls; a statistically significant difference was found at 60 minutes vs Madopar-treated subjects $(p<0.05)$. In patients washed out from Madopar for 4 days, Madopar induced a rise in plasma GH levels more delayed and sustained than that present in controls, though the overall $\mathrm{GH}$ response was not statistically different. A difference was instead present after Madopar between subjects withdrawn and on Madopar therapy $(3.1 \pm 1.2$ vs $8.1 \pm 1.7 \mathrm{ng} / \mathrm{ml}$, at 60 minutes, $\mathrm{p}<0.05$ ) (fig 1, right panel).

In a following experiment the $\mathrm{GH}$ response to acute Madopar administration was investigated in Parkinsonian subjects on Madopar or Sinemet therapy. In patients on Sinemet therapy basal GH levels were not different from those present in subjects on Madopar therapy $(1.8 \pm 0.3$ vs $0.7 \pm 0.1$ 
$\mathrm{ng} / \mathrm{ml}$ respectively). In patients medicated with Sinemet the GH-releasing effect of Madopar was markedly reduced (peak levels $4.0 \pm 2 \cdot 2 \mathrm{ng} / \mathrm{ml}$ at 60 minutes vs peak levels of $9.4 \pm 0.7 \mathrm{ng} / \mathrm{ml}$ at $90 \mathrm{~min}$ in patients on Madopar). However, at no time interval was a difference present between GH levels in the two groups (data not shown). In patients washed out from Sinemet for 4 days, there was following acute Madopar administration a trend towards higher GH levels, though values were not different from those of subjects on Sinemet therapy (data not shown).

\section{Prolactin}

There was no difference in basal PRL levels in unmedicated patients, patients on Madopar therapy and controls $(4 \cdot 9 \pm 1 \cdot 0,5 \cdot 1 \pm 1 \cdot 0,5 \cdot 4 \pm 0.6 \mathrm{ng} / \mathrm{ml}$, respectively). Figure 2 (left panel) reports the effect of Madopar in controls, unmedicated patients and patients on Madopar therapy. Madopar induced a clearcut rise in plasma PRL in either group (111 \pm $5 \cdot 0,73 \pm 13 \cdot 0,68 \cdot 1 \pm 18 \cdot 4 \mathrm{ng} / \mathrm{ml}$ at 90 minutes, respectively). A higher PRL response was present in controls than in unmedicated patients and patients on Madopar therapy at $150 \mathrm{~min}(65 \pm 17 \cdot 3$ vs $32 \pm$ $5 \cdot 3$ and vs $29 \pm 6 \cdot 1, \mathrm{ng} / \mathrm{ml}$ respectively, $\mathrm{p}<0.05$ ).

Figure 2 (right panel) shows that Madopar induced a different PRL response in Parkinsonian patients on Madopar or Sinemet therapy. In fact, in the latter group the PRL-releasing effect of Madopar was strikingly reduced (peak PRL levels $22 \cdot 2 \pm 2 \mathrm{ng} / \mathrm{ml}$

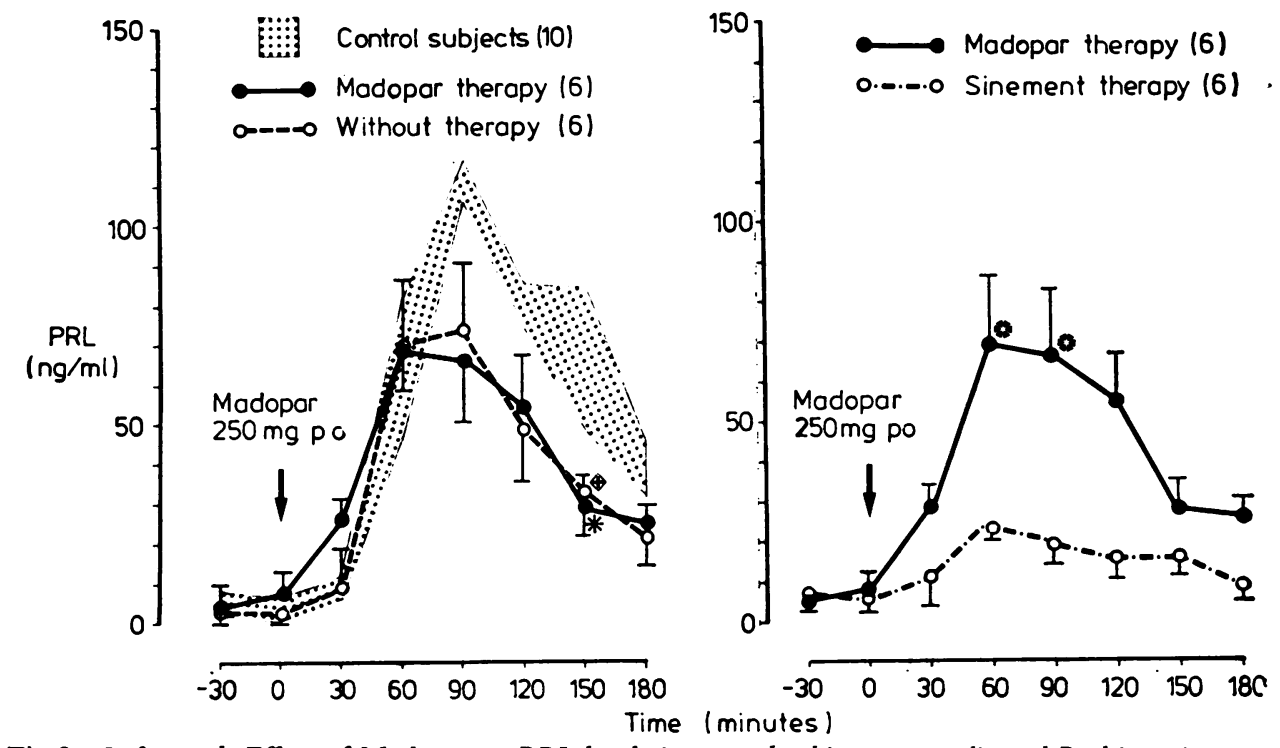

Fig 2 Left panel. Effect of Madopar on PRL levels in control subjects, unmedicated Parkinsonian subjects and patients on Madopar therapy. * = difference statistically significant between patients on Madopar therapy and controls: $\uparrow=$ difference statistically significant between unmedicated PD subjects and controls. Right panel. Effect of Madopar on PRL levels in Parkinsonian patients on Madopar or Sinemet therapy. $\mathbf{0}=$ difference statistically significant vs patients on Sinemet therapy. at 60 minutes). In patients on Sinemet regimen, a difference was present compared to values of patients on Madopar therapy at 60 and 90 minutes $(\mathrm{p}<0.05)$. In addition, PRL levels in patients on Sinemet therapy after Madopar administration were lower than in unmedicated patients given Madopar at 60 and 90 minutes $(\mathrm{p}<0.05)$ (data not shown).

Withdrawal of Sinemet resulted in a PRL response similar to that present in unmedicated or Madopartreated patients (peak PRL levels $63 \cdot 7 \pm 25 \cdot 8 \mathrm{ng} / \mathrm{ml}$ at 90 minutes), though a statistical significance vs levels of patients on Sinemet therapy was not attained (data not shown). Subjects withdrawn from Madopar were not studied.

\section{LISURIDE STUDIES}

\section{Growth hormone}

Basal GH levels in unmedicated Parkinsonian subjects were not different from those present in controls $(0.9 \pm 0.3$ vs $1.2 \pm 0.3 \mathrm{ng} / \mathrm{ml}, \mathrm{p}=\mathrm{NS})$; neither treatment with Madopar nor treatment with Sinemet affected basal GH levels $(0.7 \pm 0.2$ and $1 \cdot 0 \pm 0 \cdot 2 \mathrm{ng} / \mathrm{ml}$, respectively, $\mathrm{p}=\mathrm{NS}$ vs unmedicated subjects). Figure 3 (left panel) shows that lisuride increased plasma GH levels in controls not differently than in unmedicated subjects (peak levels $7 \cdot 0 \pm 0.8$ and $5.0 \pm 2.6 \mathrm{ng} / \mathrm{ml}$, respectively at 120 minutes). Figure 3 (right panel) shows the effect of lisuride in controls and patients on Madopar or Sinemet 


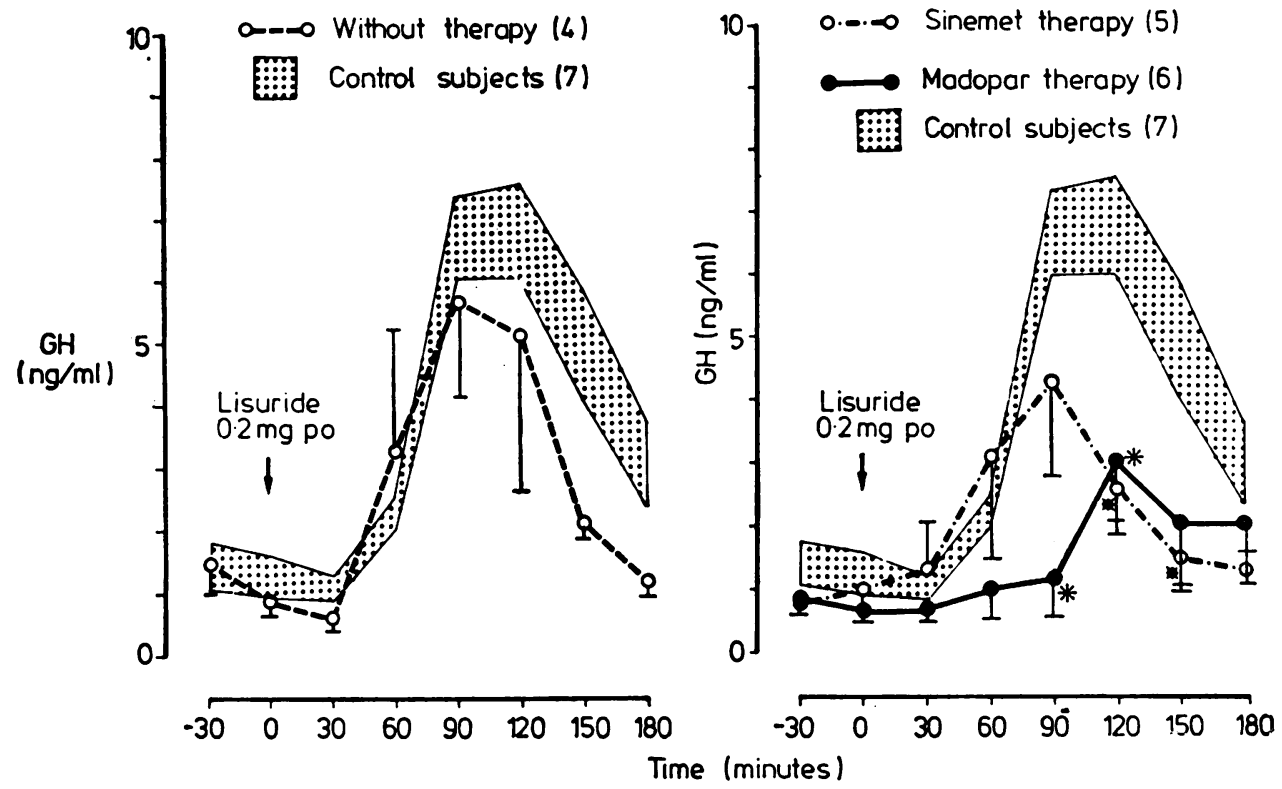

Fig 3 Left panel. Effect of lisuride on GH levels in control subjects and unmedicated Parkinsonian subjects. Right panel. Effect of lisuride on GH levels in control subjects and patients on Madopar or Sinemet therapy. * = difference statistically significant between patients on Madopar therapy and controls: $\quad=$ difference statistically significant between patients on Sinemet therapy and controls.

therapy. It is evident that in subjects receiving Madopar or Sinemet the GH-releasing effect of lisuride was considerably reduced (peak levels $3.0 \pm 0.9 \mathrm{ng} / \mathrm{ml}$ at 120 minutes and $4.0 \pm 1.5 \mathrm{ng} / \mathrm{ml}$ at 90 minutes, respectively). For patients on Madopar or Sinemet therapy there was a difference compared to control values at 90 and 120 minutes and 120 and 150 minutes, respectively.

Withdrawal of Sinemet did not alter the blunted $\mathrm{GH}$ response to lisuride; in this instance $\mathrm{GH}$ levels peaked in plasma at 90 minutes $(4.2 \pm 0.8 \mathrm{ng} / \mathrm{ml})$, with no difference being present vs values of either controls or subjects on Sinemet therapy (data not shown). Subjects withdrawn from Madopar were not studied.

\section{Prolactin}

Basal PRL levels in unmedicated patients were not different from those present in controls $(5 \cdot 2 \pm 3 \cdot 3 \mathrm{vs}$ $9.9 \pm 3.2 \mathrm{ng} / \mathrm{ml}, \mathrm{p}=\mathrm{NS}$ ); neither treatment with Madopar nor treatment with Sinemet affected basal PRL levels $(6 \cdot 1 \pm 3.1$ and $8.7 \pm 2.2 \mathrm{ng} / \mathrm{ml}$, respectively, $p=$ NS vs values of unmedicated patients and controls). Regardless of the experimental group, lisuride induced a clearcut lowering of basal PRL levels, with nadir values occurring in controls at 180 minutes $(2.3 \pm 0.9 \mathrm{ng} / \mathrm{ml})$, in unmedicated subjects at 180 minutes $(1 \cdot 1 \pm 0.4 \mathrm{ng} / \mathrm{ml})$, in Madopar-treated subjects at 180 minutes $(2 \cdot 2 \pm 0.3 \mathrm{ng} / \mathrm{ml})$, in Sinemet- treated subjects at 150 minutes $(4.0 \pm 1.4 \mathrm{ng} / \mathrm{ml})$; irêp patients withdrawn from Sinemet at 150 minutes $(3.2 \pm 0.3 \mathrm{ng} / \mathrm{ml})$ (data not shown).

\section{Discussion}

Single administration of levodopa with benserazide (Madopar) to normal subjects or to Parkinsonian subjects unmedicated or on chronic Madopar therapy induced changes in plasma GH and PRL titres different from those which might be anticipated on the basis of the mechanism of action of the single drug's components. Blockade of the conversion of administered levodopa to DA by benserazide only at the periphery 12 would result in shunting of higher amounts of unmetabolised DOPA to the CNS and thence in a greater activation of DA receptors involved in GH control. ${ }^{4}$ Contrary to their predictions, the GH response elicited by Madopar was consistently lower than that would have been anticipated after administration of levodopa alone to normal old subjects, ${ }^{12}$ and in sharp contrast with the clearcut $\mathrm{GH}$ rise present in normal individuals given the companion drug, Sinemet. ${ }^{513}$

The median eminence (ME) and the anterior pituitary (AP) are two areas unprotected by the $\mathrm{BBB} ;{ }^{14}$ therefore, our results might be attributable to a decreased availability of DA due to action of benserazide at the level of these structures. However, 
this hypothesis is difficult to reconcile with (1) the different patterning of $\mathrm{GH}$ response induced by Sinemet, for carbidopa shares the same mechanism of action of benzerazide, and ( 2 ) the evidence, though circumstantial, favouring the independence of ME$\mathrm{DA}$ neurons from $\mathrm{GH}$ regulation ${ }^{15}$ and showing the absence of DA receptors on normal somatotrophs. ${ }^{16}$

It may be proposed, alternatively, that benserazide at the dose used penetrates inside the BBB and hinders, at least partially, the conversion of levodopa to DA in areas located above the ME, thus resulting ultimately in a diminished activation of DA receptors involved in $\mathrm{GH}$ control. It must be recalled that in Madopar the ratio L-AAAD inhibitor/levodopa is $1: 4$, but it is only $1: 10$ in Sinemet; the absolute amount of benserazide contained in Madopar (50 $\mathrm{mg}$ ) is double the amount of carbidopa present in Sinemet $(25 \mathrm{mg})$. It is known that at high doses inhibitors of L-AAAD may penetrate the BBB. ${ }^{1}$

The proposition that benserazide, in this dosage contained in one Madopar capsule, may cross the BBB is also supported by data obtained in control subjects when the effect of Madopar on PRL secretion is considered. Administration of Madopar was associated with striking rises in plasma PRL, a pattern in sharp contrast with the prolactin-lowering effect of Sinemet, 1317 possibly due to benserazideinduced suppression of DA function inside the ME. It is noteworthy that benserazide alone reportedly induces in normal subjects a rise in plasma PRL starting at the low dose of $10 \mathrm{mg}$ orally, while the minimal dose of carbidopa effective in this respect is $80 \mathrm{mg} .{ }^{18}$ Thus, the minimal effective dose for benserazide is lower, and for carbidopa higher, than the amount of each compound present in the two anti-parkinsonian drugs, a fact which may account for the different patterning of the endocrine response to Sinemet and Madopar.

Granted that benserazide may partially cross the BBB, let us consider how this proposition may explain the endocrine responses to Madopar of Parkinsonian subjects. In patients on Madopar therapy, Madopar elicited a GH response which was greater than that it induced in controls. On pursuing the same type of reasoning, this may be related either to a lower penetration of benserazide into the CNS of Parkinsonian patients or, alternatively and more likely, to the DA formed from levodopa acting on more sensitive hypothalamic(?) DA receptors. The finding that in unmedicated Parkinsonian patients or in patients withdrawn from therapy the GH response to Madopar was similar to that present in controls would indicate that the patterning of $\mathrm{GH}$ response to acute Madopar administration in Parkinsonian subjects is best explained by chronic drug exposure, and not by an inherent hypersensi- tivity of DA receptors related to $\mathrm{GH}$ control. The peculiar effect of chronic exposure to Madopar on the DA system for GH control seems supported by the finding that in subjects on Sinemet therapy, the GH-releasing effect of Madopar was blunted. It is tempting to speculate that enhancement of DA tone at hypothalamic DA receptors by combined levodopa-carbidopa administration ${ }^{13} 1720$ would reduce responsiveness to a subsequent DA-mediated stimulus such as that of Madopar. However, since patients on chronic Sinemet therapy were about 15 years older than those on chronic Madopar therapy, the possibility cannot be excluded that in the former group the blunted GH response to Madopar may be related to the different age. 1221

Evaluation of plasma PRL responses to Madopar in PD subjects unmedicated or on Madopar therapy, and their comparison with the PRL response to Madopar of controls, showed some differences. While the PRL response of patients on Madopar therapy could not be discriminated from that of unmedicated patients, these two responses were lower than that evoked by Madopar in controls. Blunting of the PRL-releasing effect of Madopar in unmedicated Parkinsonian subjects is difficult to interpret, although in subjects on Madopar therapy it may reflect the presence of a reduced tuberoinfundibular sensitivity to DA. Interestingly, a blunted PRL-releasing effect is present after benserazide in subjects harbouring a PRL-secreting pituitary tumour, ${ }^{22}$ in whom proof has been provided for a defective hypothalamic DA function. ${ }^{172324}$ The finding that there was a striking reduction of the PRL-releasing effect of Madopar in subjects on Sinemet therapy may be explained by a replenishment of tubero-infundibular DA stores by Sinemet, overriding the ability of acute Madopar administration to impair hypothalamic DA tone. Other possibilities, however, cannot be excluded; thus, reduced PRL response to Madopar in subjects on Sinemet therapy, and also in unmedicated subjects, may be due to reduction of the pituitary pool of PRL as a consequence of sustained enhancement of tuberoinfundibular DA function. In this context it is worth noting that the PRL response to thyrotropin releasing hormone (TRH), which is under inhibition by dopmainergic tone, ${ }^{25}$ is lower in unmedicated Parkinsonian subjects and in subjects on chronic Sinemet therapy than in subjects on chronic Madopar therapy (A. Martinez-Campos, unpublished observations).

Whatever answers will finally be given to these questions, the specific involvement of previous Sinemet therapy is shown by the fact that its withdrawal resulted in a PRL response similar to that present in unmedicated or Madopar-treated patients. At odds with our conclusion that in unmedicated 
patients hypothalmic DA function seems to be preserved is that of Lawton and MacDermot. ${ }^{26}$ These authors, on the basis of studies conducted with TRH and Sinemet, have suggested that the DA defect in idiopathic Parkinson's disease extends to the hypothalamus and is not confined to the basal ganglia.

The bulk of data obtained in the Madopar studies were compatible with the idea that chronic administration of levodopa and benserazide, with the latter probably able to penetrate partly into the CNS, engenders a state of relative denervation supersensitivity ${ }^{27}$ of hypothalamic DA neurons. If this were the case, administration of a direct DA receptor agonist should evoke a greater $\mathrm{GH}$ response in subjects on Madopar therapy than in Sinemetpretreated, unmedicated Parkinsonian, or control subjects. PRL secretion, instead, should be suppressed in all experimental groups, due to the ability of DA receptor agonists to stimulate DA receptors located on the pituitary lactotrophs. ${ }^{28}$

This was the reason for using the direct DA receptor agonist, lisuride. ${ }^{10}$ However, irrespective of the type of preceding therapy, lisuride administered to Parkinsonian subjects on Madopar or Sinemet induced a small GH rise, which was lower than that elicited in controls. No difference was present in the GH response to lisuride between unmedicated and control subjects, a fact which reinforces the idea that hypothalamic DA function related to GH control is preserved in unmedicated Parkinsonian subjects.

The blunted $\mathrm{GH}$ response to lisuride in patients on Sinemet is reminiscent of the poor GH-releasing effect of Madopar in the same patients and may have a similar interpretation (see above). However, at odds with the expectations, the same response pattern was present in subjects on Madopar therapy, in whom a higher GH responsiveness to DA stimulation might have been anticipated. No reason is available to explain this discrepancy; certainly, the GH-releasing effect of orally-administered lisuride also was small in control subjects in agreement with the low $\mathrm{GH}$ response to DA stimulation of adult women. ${ }^{12} 2129$ This fact prevented a clearcut differentiation in GH responses among different experimental groups. It is also difficult to explain the finding that withdrawal of Sinemet did not alter the sluggish GH response to lisuride, whereas in the Madopar studies withdrawal of Sinemet had been found to restore the original hormone responsiveness.

That chronic treatment with inhibitors of LAAAD may have increased the metabolism of lisuride thus causing diminished circulating drug levels, is negated by the finding that the PRLlowering effect of lisuride was equally manifested in all groups. Lisuride's inability to demonstrate hyperresponsiveness of DA receptors for $\mathrm{GH}$ control in Parkinsonian subjects on Madopar, may be due to this ergoline acting on a DA receptor different from that acted upon by DA generated via conversion from levodopa. ${ }^{31}$ Certainly, further studies on a more extensive series of patients, with the use of other routes of administration ${ }^{32}$ and additional DA agonists such as bromocriptine, are necessary to resolve this problem.

In summary, it would appear from these studies that Madopar given chronically to Parkinsonian subjects may impair the activity of DA function for GH and PRL control, an effect probably due to penetration of benserazide across the blood brain barrier in the region of the hypothalamus. If the same were true of the blood brain barrier surrounding the nigrostriatal system, Madopar would be ineffective in the treatment of Parkinsonism. However, since Madopar and Sinemet are equally effective in relieving the impaired DA function in these extrapyramidal centres, ${ }^{19}$ it is suggested that the different properties of the blood brain barrier hinder penetration of benserazide at this level. Demonstration of supersensitivity of hypothalamic DA neurons implicated in GH control was not achieved in Parkinsonian subjects on Madopar therapy challenged with lisus ride. It is at present unknown whether this is due top lack of denervation supersensitivity in DA neurons for $\mathrm{GH}$ control, ${ }^{32}$ or to an inappropriate use of lisuride as the prototype of a direct DA agonisto Another conclusion generated from this study is that, apart from their use in association to levodopæa in the treatment of Parkinsonism, "peripheral'? inhibitors of L-AAAD, and notably benserazide, are useful tools for investigating DA function in the hypothalamus of neurological and endocrinological patients. ${ }^{22}$

We are grateful to Dr Daniela Cocchi for active participation in the initial part of these studies and to Miss Isabella Zago for secretarial help.

\section{References}

${ }^{1}$ Porter J. Aromatic aminoacids and decarboxylase inhibitors. Fed Proc 1971;30:871-6.

${ }^{2}$ Bartholini G, Pletscher A. Decarboxylase inhibitors. Pharmacol Ther (B) 1975;3:407-21.

${ }^{3}$ Lakke JPWF, Korf J, Wesseling H. (eds) Parkinson's Disease. Concepts and Prospects. Amsterdam: Excerpta Medica, 1977.

${ }^{4}$ Müller EE, Nisticò G, Scapagnini U. Neurotransmitters and Anterior Pituitary Function. New York: Academic Press, 1978. 
5 Mars H, Genuth SM. Potentiation of levodopa stimulation of human growth hormone by systemic decarboxylase inhibition. Clin Pharmacol Ther 1973; 14:390-5.

${ }^{6}$ Schömberger W, Grimm W, Ziegler R. The effect of Nacom (L-dopa and L-carbidopa) on growth hormone secretion in 75 patients with short stature. Europ J Pediatr 1977;127:15-19.

7 Brown GM, Garfinkel PE, Warsh JJ, Stancer HC. Effect of carbidopa on prolactin, growth hormone and cortisol secretion in man. J Clin Endocrinol Metab 1976;43:236-9.

${ }^{8}$ Ruggieri S, Falaschi P, Baldassarre M. D'Orso R, Frajese S, Agnoli A. Neuroendocrine response to active drugs in Parkinson's disease. In: Müller EE, Agnoli A, eds. Neuroendocrine Correlates in Neurology and Psychiatry. Amsterdam: Elsevier/NorthHolland, 1979:127-37.

9 Pontiroli AE, Castegnaro E, Vettaro MP, Viberti CC, Pozza G. Stimulatory effect of the dopa decarboxylase inhibitor Ro 4-4602 on prolactin release: inhibition by L-DOPA, metergoline, methysergide and 2- $\beta$ - $\alpha$-ergocriptine. Acta Endocrinol (Kbh) 1977; 84:36-44.

${ }^{10}$ Horowski R, Neumann F, Graf KJ. Effect of apomorphine hydrocloride, dibutyril-apomorphine and Lysenil on plasma prolactin concentrations in the rat. J Pharm Pharmacol 1975;27:533-4.

11 Dunnett CW. New tables for multiple comparisons with a control. Biometrics 1964;20:482-92.

12 Sachar EJ, Mushrush G, Perlow M, Weizman ED, Sassin J. Growth hormone response to L-Dopa in depressed patients. Science 1972;173:1304-5.

${ }^{13}$ Frantz AG, Suh HK, Noel GL. Effects of l-dopa on prolactin secretion in humans. In: Usdin E, Snyder $\mathrm{SH}$, eds. Frontiers in Catecholamine Research. New York: Pergamon Press, 1973:843-6.

14 Weindl A, Joynt RJ. The median eminence is a circumventricular organ. In: Knigge KM, Scott DE, Weindl A, eds. Brain-endocrine interaction. Median Eminence: structure and function. Basle: Karger, 1972:280-97.

${ }^{15}$ Casanueva F, Betti R, Cocchi D, Zanardi P, Motta T, Müller EE. A role for dopamine in growth hormone regulation in the dog. Endocrinology 1981;108: 1469-75.

${ }^{16}$ Müller EE, Salerno F, Cocchi D, Locatelli V, Panerai AE. Interaction between the thyrotropin-releasing hormone-induced growth hormone rise and dopaminergic drugs: studies in pathologic conditions of the animal and man. Clin Endocrinol 1979;11:64556.

17 Fine SA, Frohman LA. Loss of central nervous system component of dopaminergic inhibition of prolactin secretion in patients with prolactin-secreting pituitary tumors. J Clin Invest 1978;61:973-80.

18 Polleri A, Masturzo P, Murialdo G, Carolei A. Dose and sex related effects of aromatic amino acids decarboxylase inhibitors on serum prolactin in humans. Acta Endocrinol (Kbh) 1980;93:7-12.

19 Rinne UK, Molsa P. Levodopa with benserazide or carbidopa in Parkinson disease. Neurology (Minneap) 1979;29:1584-9.

${ }^{20}$ Woolf PD, Leebaw WF, Lee LA. Effect of CNS dopamine augmentation on stimulated prolactin secretion. J Clin Endocrinol Metab 1977;45:857-60.

${ }^{21}$ Maany I, Frazer A, Mendels J. Apomorphine: effect on growth hormone. J Clin Endocrinol Metab 1975; 40:162-3.

22 Pontiroli AE, Falsetti L, Voltolini AM, et al. Pozza G.R04-4602, a stimulator of prolactin (PRL) release: pharmacologic interactions and diagnostic application in Hyper PRL patients. In: Pecile A, Müller EE, eds. Abstract Book Int Symp Growth Hormone and Other Biologically Active Peptides. Ricerca Scientifica ed Educazione Permanente, 1979:80.

${ }^{23}$ Van Loon GR. A defect in catecholamine neurons in patients with prolactin-secreting pituitary adenomas. Lancet 1979;2:868-71.

${ }^{24}$ Müller EE, Genazzani AR, Murru S. Nomifensine: diagnostic test in hyperprolactinemic states. $J$ Clin Endocrinol Metab 1978;47:1352-7.

${ }^{25}$ Refetoff S, Fang VS, Rapaport B, Friesen HG. Interrelationships in the regulation of TSH and prolactin secretion in man: effects of L-Dopa, TRH and thyroid hormone in various combinations. $J$ Clin Endocrinol Metab 1974;38:450-7.

${ }^{26}$ Lawton NP, MacDermot J. Abnormal regulation of prolactin release in idiopathic Parkinson's disease. J Neurol Neurosurg Psychiatry 1980;43:1012-5.

27 Gianutsos G, Drawbaugh RB, Hynes MD, Lal H. Behavioral evidence for dopa-minergic supersensitivity after chronic haloperidol. Life Sci 1974;14: 887-98.

${ }^{28}$ MacLeod RM. Regulation of prolactin secretion. In: Martini L, Ganong WF, eds. Frontiers in Neuroendocrinology, Vol IV. New York: Raven Press, 1976: 169-94.

${ }^{29}$ Ettigi P, Lal S, Martin JB, Friesen HG. Effect of sex, oral contraceptives and glucose loading on apomorphine-induced growth hormone secretion. J Clin Endocrinol Metab 1975;40:1094-8.

${ }^{30}$ Silbergeld EK, Hruska RE. Lisuride and LSD: Dopaminergic and serotoninergic interactions in the serotonin syndrome. Psychopharmacology 1979;65: 233-7.

${ }^{31}$ Schachter $M$, Bedard $P$, Debono AG, Jenner $P$. Marsden CD, Price P. Parkes JD, Keenan J, Smith B, Rosenthaler J, Horowski R, Dorow R. The role of D-1 and D-2 receptors. Nature 1980;286:157-9.

32 Lal S, Nair NPV. Growth hormone and prolactin responses in neuropsychiatric research. In: Müller EE, Agnoli A, eds. Neuroendocrine Correlates in Neurology and Psychiatry. Amsterdam: Elsevier/ North-Holland, 1979:179-94. 